\title{
Deprivation, Healthcare Accessibility and Satisfaction: Geographical Context and Scale Implications
}

\author{
Pablo Cabrera-Barona ${ }^{1}$ - Thomas Blaschke ${ }^{1}$. \\ Gabriel Gaona ${ }^{2}$
}

Received: 21 July 2016 / Accepted: 31 January 2017 /

Published online: 1 March 2017

C The Author(s) 2017. This article is published with open access at Springerlink.com

\begin{abstract}
Indices explaining health phenomena are important tools for identifying and investigating health inequalities and to support policy making. Some of these indices are expressed at area-level, and the investigation of the areal influences of these indices on individual health outcomes have scale and geographical contextual implications that need to be assessed. In this study we calculated two area-level indices: one deprivation index and one index of healthcare accessibility. Using multilevel modelling, we calculated the area-level influences of these indices on an individual-level index of healthcare satisfaction considering three kinds of areas or contexts: a context of deprivation, a context of healthcare accessibility and a context combining the two characteristics of healthcare accessibility and deprivation. We evaluated two kinds of geographical problems using the statistical results of these area-level influences: the modifiable areal unit problem (MAUP) and the uncertain geographic context problem (UGCoP). Regarding the MAUP we evaluated the scale effects at two scales: census blocks and census tracts. Regarding the UGCoP we evaluated the differences in areal influences between the three kinds of contexts for both scales. The case study area was the city of Quito, Ecuador. The results of the performed analyses showed no severe MAUP and UGCoP, and revealed important evidence of the area-level influence of deprivation and healthcare accessibility on healthcare satisfaction.
\end{abstract}

Pablo Cabrera-Barona

pablo.cabrera-barona@stud.sbg.act.at

Thomas Blaschke

thomas.blaschke@sbg.ac.at

Gabriel Gaona

gabriel.gaona@ikiam.edu.ec

1 Interfaculty Department of Geoinformatics - Z_GIS, University of Salzburg, Schillerstraße 30, 5020 Salzburg, Austria

2 Universidad Regional Amazónica IKIAM, Vía Muyuna, Kilómetro 7, Tena, Ecuador 
Keywords Modifiable areal unit problem (MAUP) - Uncertain geographic context problem $(\mathrm{UGCoP}) \cdot$ Neighborhood effects · Deprivation · Healthcare accessibility Healthcare satisfaction

\section{Introduction}

The choice of the reporting units for communicating health-related information has methodological and practical implications for information users (Hagenlocher et al. 2014; Nelson and Brewer 2015). Health analyses with implications in decision making have widely used measures of healthcare accessibility and social deprivation (Bell et al. 2007; Bissonnette et al. 2012; Boyle et al. 2001; Cabrera-Barona et al. 2015; Carstairs 1995; Crooks and Schuurman 2012; Delamater 2013; Havard et al. 2008; Hiscock et al. 2008; Lalloué et al. 2013; Luo 2014; Wan et al. 2013). There are different indicators that can be used to represent deprivation and health. Townsend et al. (1988) demonstrated a strong association between health indicators and deprivation indicators and also between a composite health index and a composite deprivation index. Composite indices are measures constructed by aggregating different indicators, and represent multidimensional phenomena, i.e. healthcare accessibility or deprivation. Several studies analyze the effects on health with simple indicators, and little attention has been paid to the analyses of health effects using composite indices. This study does not use simple indicators, but rather composite indices: two area-level indices (one index of deprivation and one of healthcare accessibility) and one individual-level index of healthcare satisfaction.

The term deprivation is used to name composite indices representing social and material disadvantages (Townsend 1987). These indices are useful for identifying inequalities in health outcomes (Cabrera-Barona et al. 2015; Boyle et al. 2001; Havard et al. 2008; Pampalon et al. 2009; Townsend 1987). Because deprivation indices are commonly based on census areas, these indices are also place-specific measures related to health (Bell et al. 2007).

Assessing the access to primary healthcare services is fundamental for decision making in the operation of a health system. Accessibility to healthcare services can be evaluated considering geographical distances between people's households and healthcare services and also considering the healthcare opportunities in terms of the number of healthcare services a person can choose from (Delamater 2013; Penchansky and Thomas 1981). This combination of geographical access and healthcare opportunities is called spatial accessibility (Guagliardo 2004) and can be calculated using a gravity-based model, which is based on the number of opportunities (e.g. number of healthcare services), distances to these opportunities, and a gravity decay coefficient representing the distance decay (Hansen 1959).

The measures of healthcare accessibility derived from gravity-based models are measures of potential physical accessibility to healthcare. Joseph and Phillips (1984) suggested that these measures of potential accessibility to healthcare are easy to interpret, but that it is also important to evaluate the realized access to healthcare. The realized access to healthcare is equivalent to the use of healthcare services. The use of healthcare services can be interpreted using a specific health outcome: the satisfaction of healthcare services users (Andersen 1995). Additionally, a population's 
characteristics (e.g. the population's levels of deprivation) are also factors influencing healthcare satisfaction (Aday and Andersen 1974; Andersen 1995).

The healthcare satisfaction is the patient's evaluation of the received healthcare service (Pascoe 1983), and can be evaluated by measuring the patient's reported experience of the healthcare service, as well as by some characteristics of the service, e.g. number of physicians in service (Pascoe 1983; Bleich et al. 2009; Hekkert et al. 2009).

Deprivation can be associated with healthcare accessibility and with healthcare outcomes (Butler et al. 2013). Although individual-level characteristics have an influence on healthcare satisfaction (Hekkert et al. 2009), analyses of public health must also focus on the issue of place-related health. The place or local neighborhood influences health outcomes. The neighborhood is also referred to as the context of the individuals, and, from a geographical perspective, it can be represented in welldefined areas such as census blocks. The context can be represented by socio-economic features and access to urban facilities.

A key issue arises from the presented concepts: the contexts of deprivation and healthcare accessibility are likely to influence an individual-level health outcome such as healthcare satisfaction. To represent these contexts we used the two area-level indices of deprivation and healthcare accessibility. The area-level influence of these indices on healthcare satisfaction can be studied, to better understand the place-people dynamics of public health, with important implications in policy making. The geographical variations in health exist at many scales, from global to local (Boyle et al. 2001). Neighborhoods or contexts to express health-related measures can be defined in different ways. However, the most practical way is to use census areas such as census blocks and census tracts (Flowerdew et al. 2008).

Despite the important role that the area-based measures of deprivation and healthcare accessibility play for health policy making, little attention has been paid to evaluating the contextual and scale implications of these measures, and only few studies have addressed this issue (Cabrera-Barona et al. 2016b; Dumedah et al. 2008; Haynes and Gale 2000; Schuurman et al. 2007; Wei et al. 2017). The phenomenon of the modifiable areal unit problem (MAUP) usually causes the results of statistical analyses to differ according to the scale and size of the spatial reporting units (Briant et al. 2010; Openshaw 1984). The effects of MAUP have been analyzed using several approaches, generally based on correlation and regression methods (Flowerdew 2011; Fotheringham and Wong 1991; Openshaw 1984; Pietrzak 2014; Soobader et al. 2001), and these kinds of analyses have typically been applied to census indicators (Flowerdew 2011; Soobader et al. 2001).

There is another kind of problem less discussed than MAUP: the uncertain geographic context problem (UGCoP) identified by Kwan (2012a). This problem refers to the uncertainty of the effects of area-based measures due to the different delineation of the units of analysis, and to the uncertainty of the deviation from the relevant geographic context that is, in principle, unknown (Diez-Roux and Mair 2010). These uncertainties are spatial but also temporal.

In the case of the UGCoP, different kinds of spatial units influence individual-level outcomes in different ways, while in the case of the MAUP, different configurations of the spatial units causes different aggregation levels of the individuals, which means different statistical results. For this reason, the zoning effect in the MAUP is also known as the aggregation effect when changing the divisions of a study area, even at the same scale. 
The other UGCoP uncertainty is related to the time mismatches that can occur when relating the individuals with their geographical contexts. In other words, the individuals' dynamics are very complex and the characteristics of these individuals are not only influenced by contextual effects of the neighborhoods where they live, but also by other neighborhoods that they visit at different times (Kwan 2012a).

In general, these spatial and temporal uncertainties have been identified when areabased measures (e.g. deprivation) were used to explain individual behaviors or outcomes (e.g. satisfaction with healthcare) (Kwan 2012b). After defining the nature of the contexts and the individuals, the contextual effects on health outcomes can be evaluated using multilevel models (Kwan 2012a; Kwan 2012b).

The UGCoP is especially relevant in this study because healthcare satisfaction is measured considering people's perceptions out-of-healthcare services. To create the index of healthcare satisfaction we used data extracted from interviews conducted in households. We thus have a measure of actual access to healthcare in a context of potential spatial access to healthcare. Additionally, the unknown optimal spatial configuration of deprivation areas could differ from the spatial configuration of census blocks and census tracts traditionally used to express deprivation.

Based on the concepts and approaches presented, the overall goal of this research is to analyze whether important scale effects of MAUP and effects of the UGCoP exist when analyzing the influence of area-based measures of deprivation and healthcare accessibility on individual healthcare satisfaction at two different scales: census blocks and census tracts. We also aim to evaluate the area-level influence of deprivation and healthcare accessibility on healthcare satisfaction.

A census tract is the geographical unit created by aggregating census blocks. The capital city of Ecuador, Quito, is used as the case study area. The remainder of the paper is organized as follows: the next section explains how the health-related indices were constructed. In the same section, the multilevel modelling used in the analyses is explained. The outcomes of the indices calculations and the findings of the multilevel calculations are shown in the results section. The paper concludes with a discussion including decision making implications, importance of the findings, limitations of the study and future research directions.

\section{Methods}

Two area-level indices were calculated: a deprivation index and an index of healthcare accessibility. These two area-level indices were calculated at two different scales: census blocks and census tracts. An individual-level index of healthcare satisfaction was also calculated. The area-level influences of deprivation and healthcare accessibility on healthcare satisfaction were calculated using multilevel models. We used these calculations to evaluate the MAUP and the UGCoP.

\section{Calculation of the Deprivation Index}

When constructing deprivation indices, there are methodological and operative issues that need to be considered, such as possible outliers in the data, weighting techniques or appropriate census measures (Senior 1991). To develop the deprivation index a multi- 
criteria decision analysis approach (Boroushaki and Malczewski 2008; Saaty 1977) was applied, and the guidelines proposed by the Organisation for Economic Co-operation and Development (OECD 2008) for constructing an index were adhered to.

Ten indicators were used to compose the deprivation index. These indicators were extracted from the 2010 Ecuadorean Population and Housing Census, represent social and material disadvantages in Quito, and can be related to health issues (CabreraBarona et al. 2016b). The conceptual reference for selecting the indicators used to construct the deprivation index (Table 1) is based on the human-rights approach of the good living standards (Cabrera-Barona et al. 2015; Mideros 2012). A good living that includes a healthy life is achieved when basic human rights are ensured through the access to basic services and welfare conditions, such as access to clean water or education. When access to these services is limited, then people have a lower living standard, and are thus more deprived. Additionally, the chosen indicators have an affinity with the material and social dimensions that can be related to health (Carstairs 1995; Pampalon et al. 2009; Pasetto et al. 2010; Townsend 1987). To detect possible multicollinearities of the indicators, the variance inflation factors (VIFs) between indicators were calculated (OECD 2008). All the VIFs obtained were lower than five and we can therefore confirm that all ten indicators can be used to construct the deprivation index.

The indicators composing a deprivation index need to be weighted in order to assign relative importance to the different conditions represented by these indicators. A common method to calculate weights of indicators of a deprivation index is principal components (Pampalon et al. 2009). However, this method does not reflect knowledge and judgments from experts and policy makers. An alternative method is the analytical hierarchy process (AHP, Saaty 1977). The AHP is a multi-criteria decision analysis method that supports the creation of weights based on experts' judgments, and evaluates the reliability of these weights by applying a consistency test. The key aspect of the AHP is the construction of a pairwise comparison matrix (Boroushaki and Malczewski 2008; Saaty 1977; Saaty 1987). This is a $\mathrm{n} \times \mathrm{n}$ matrix where $n$ is the number of indicators and its inputs are the Saaty's scales (Table 2) and can be defined as a matrix

Table 1 Indicators used to construct the deprivation index

Indicators
A: percent of the population that have a long-term disability (for more than one year)
$\mathrm{B}$ : percent of the population that does not have any level of formal education or instruction
$\mathrm{C}$ : percent of the population that has no public social/health insurance
D: percent of the population that works in unpaid jobs
E: percent of households with four or more persons per dormitory
F: percent of households without access to drinking water from the public system
G: percent of households without access to the sewerage system
$\mathrm{H}$ : percent of households without access to the public electricity grid
I: percent of households without garbage collection service
$\mathrm{J}$ :distance (meters)to the nearest primary healthcare service 
Table 2 Saaty's scales for the pairwise comparison matrix

\begin{tabular}{ll}
\hline Scales (intensity of importance) & Description \\
\hline 1 & Equal importance \\
3 & Moderate importance \\
5 & Strong importance \\
7 & Very strong importance \\
9 & Extreme importance \\
\hline
\end{tabular}

$X$ with the elements $a_{i j}$ (Chen et al. 2010):

$$
X=\left[a_{i j}\right], i, j=1,2,3, \ldots, n
$$

The matrix has the property of reciprocity:

$$
a_{i j}=\frac{1}{a_{i j}}
$$

The matrix is normalized and the weights of the indicators are computed as:

$$
w_{i}=\frac{\sum_{j=1}^{n} b_{i j}}{\sum_{i=1}^{n} \sum_{j=1}^{n} b_{i j}}, i, j=1,2,3, \ldots, n
$$

The term $b_{i j}$ represents the elements of the normalized matrix.

The scales are obtained from experts' interviews. Experts evaluate the contribution of all the indicators to the deprivation index by comparing them to each other and assigning values from Saaty's scales to the comparisons. For instance, one expert can conclude that having no access to clean water is very strongly more important than not having insurance. In this example, the scale of 7 is assigned (Table 2). Intermediate values of the scales $(2,4,6,8)$ can been used to represent "thresholds" judgments, and reciprocals (inverse values) of the scales can also be used. For this study we consulted 13 experts of the fields of geography, health and urban studies.

As was mentioned before, one of the advantages of the AHP is to test the consistency of the obtained weights. This test is performed by calculating a consistency index (CI) and a consistency ratio (CR). The CI is calculated with the equation (Saaty 1977):

$$
C I=\frac{\lambda_{\max }-n}{n-1}
$$

Where $\lambda_{\max }$ is a function of the weights' vector and the pairwise comparison matrix. The CR evaluates the likelihood that the judgments were obtained randomly (Saaty 1977):

$$
C R=\frac{C I}{R I}
$$


The random index (RI) is the consistency index of a randomly generated matrix and depends of the number of indicators used (Cabrera-Barona et al. 2015; Boroushaki and Malczewski 2008). In this case a value of 1.49 was assigned to RI (Boroushaki and Malczewski 2008) because ten indicators were used to construct the deprivation index. If the $\mathrm{CR}$ is lower than 0.10 then it can be concluded that the weights calculated are consistent, thus the experts' judgments are consistent.

Table 3 shows the pairwise comparison matrix, the obtained weights for each indicator, and the calculated CR. The capital letter representing each indicator is equivalent to the capital letters assigned for each indicator in Table 1.

The indicators were standardized by applying a min-max normalization to render them comparable (OECD 2008):

$$
v_{i \text { norm }}=\frac{v_{i}-v_{\min }}{v_{\max }-v_{\min }}
$$

Where $v_{i}$ is the value of one indicator in a unit area $i$, and $v_{\text {min }}$ and $v_{\max }$ refers to the minimum and maximum values of the indicator respectively.

Finally, a weighted additive aggregation (OECD 2008) of all the ten indicators was applied to each of the census areas:

$$
D_{i}=\sum v_{i \text { norm }}{ }^{*} w_{i}
$$

Where $D_{i}$ represents the deprivation index in a census area $i$, and $w_{i}$ represents the weight of the indicator $v_{i}$ norm. To facilitate interpretation, the deprivation index was normalized using the min-max normalization. Values closer to 1 represent higher deprivation.

\section{Calculation of the Index of Healthcare Accessibility}

The index of healthcare accessibility was constructed by integrating concepts of the Huff model (Huff 1963) and gravity models (Hansen 1959; Crooks and Schuurman 2012; Guagliardo 2004).

\begin{tabular}{|c|c|c|c|c|c|c|c|c|c|c|c|}
\hline Indicator & A & B & $\mathrm{C}$ & $\mathrm{D}$ & E & F & G & $\mathrm{H}$ & I & $\mathrm{J}$ & Weights \\
\hline A & 1 & & & & & & & & & & 0.048 \\
\hline B & 3 & 1 & & & & & & & & & 0.067 \\
\hline $\mathrm{C}$ & 3 & 2 & 1 & & & & & & & & 0.090 \\
\hline D & 2 & 2 & 2 & 1 & & & & & & & 0.111 \\
\hline E & 1 & 1 & $1 / 2$ & $1 / 2$ & 1 & & & & & & 0.039 \\
\hline $\mathrm{F}$ & 4 & 4 & 3 & 3 & 6 & 1 & & & & & 0.228 \\
\hline G & 2 & 2 & 1 & 1 & 4 & $1 / 2$ & 1 & & & & 0.102 \\
\hline $\mathrm{H}$ & 2 & 1 & 2 & 1 & 4 & $1 / 3$ & 2 & 1 & & & 0.108 \\
\hline I & 1 & 1 & 1 & $1 / 2$ & 3 & $1 / 3$ & 1 & 1 & 1 & & 0.076 \\
\hline $\mathrm{J}$ & 2 & 2 & 1 & 1 & 3 & 1 & 1 & 2 & 2 & 1 & 0.131 \\
\hline $\mathrm{CR}=0.03$ & & & & & & & & & & & \\
\hline
\end{tabular}

Table 3 Pairwise comparison matrix, indicators' weights, and the CR of the weights 
The accessibility in a unit area $i$ (e.g. a census block) can be expressed in the following generalized measure of accessibility (Hansen 1959):

$$
A_{i}=\sum_{j} S_{j} f\left(c_{i j}\right)
$$

Where $S_{j}$ refers to the opportunities in the area $j$, and $f\left(c_{i j}\right)$ represent a function of travel cost from unit area $i$ to area $j$.

The proposal of Hansen (1959) can be expressed with the following formula (Guagliardo 2004):

$$
A_{i}=\sum_{j} \frac{S_{j}}{d_{j}^{\beta}}
$$

The term $d_{j}^{\beta}$ is the function of travel cost. $d_{j}$ is the Euclidean distance from $i$ to $j$ and $\beta$ is the gravity decay coefficient. The $\beta$ value can be defined considering that the use of healthcare services is inversely proportional to the distance $d_{j}$ (Schuurman et al. 2010). In this case $\beta=-1$ and the travel cost function $d_{j}^{\beta}$ is then expressed $\frac{1}{d_{j}}$. In the case of healthcare accessibility the opportunities are known as a supply capacity $S_{j}$ that can be represented by different kinds of indicators, e.g. number of beds or number of healthcare professionals. For this study, the indicator available and chosen to represent $S_{j}$ was the number of healthcare professionals in the primary healthcare service.

The gravity-based equation above is only adjusted to supply and not to demand. To correct this problem Joseph and Bantock (1982) proposed the incorporation of population demand, understood as the sum of the populations that may access healthcare services, who are also affected by the travel cost function:

$$
D_{j}=\sum_{k} P_{k} * \frac{1}{d_{k j}}
$$

We used geographic information systems to define which populations to include in the equation above, and analyzed the distances from each census block centroid to the nearest primary healthcare service. We found that the average distance was $668 \mathrm{~m}$, and that most of the centroids were located less than $1000 \mathrm{~m}$ from the nearest primary healthcare service. With these criteria we defined a maximum threshold of $1200 \mathrm{~m}$ and additional thresholds of 300 and $600 \mathrm{~m}$. The population demand is then formulated as follows:

$$
D_{j}=\sum_{k \in\left\{d_{k j}<300\right\}} P_{k} \frac{1}{300}+\sum_{k \in\left\{d_{k j}<600\right\}} P_{k} \frac{1}{600}+\sum_{k \in\left\{d_{k j}<1200\right\}} P_{k} \frac{1}{1200}
$$

Adjusting the simple gravity model to the population factor, and using the travel cost function considering the maximum distance threshold, we obtained the following improved gravity model:

$$
A_{i}=\sum_{j} \frac{S_{j} * \frac{1}{1200}}{D_{j}}
$$


The improved gravity model does not consider competition among the different primary healthcare services, and, consequently, its use can lead to an overestimation of the accessibility of some services (Luo 2014). To overcome this limitation, we added the probability of people's selection of a healthcare service out of other available services to the gravity model. This probability is known as the Huff model (Huff 1963) and for our study can be expressed as follows:

$$
\operatorname{prob}_{i}=\frac{S_{j} \frac{1}{1200}}{\sum_{s \in M} S_{s} \frac{1}{1200}}
$$

Where $\operatorname{prob}_{i}$ is the probability of population in location $i$ visiting a primary healthcare service $j . S_{s}$ represents the number of healthcare professionals of the primary healthcare services found inside the maximum distance threshold of $1200 \mathrm{~m}$ from a population's location.

The last step of the proposed method of measuring healthcare accessibility is to join the improved gravity-based model and the Huff model to create the index of healthcare accessibility:

$$
A_{i}=\sum_{j} \frac{S_{j} * \frac{1}{1200}}{D_{j}} * \text { prob }_{i}
$$

The index was normalized using min-max normalization. Values closer to 1 represent higher accessibility. The calculation of the index of healthcare accessibility was automatized by a tool generated with Python 2.7, using the packages for scientific computing NumPy, ArcPy and OS.

\section{Calculation of the Index of Healthcare Satisfaction}

We used the index of composite healthcare satisfaction proposed by Cabrera-Barona et al. (2016a). This index uses three indicators: waiting time to receive healthcare attention after arriving at the primary healthcare service $(T)$, the quality of the attention received, as reported by the patient $(Q)$, and the opportunities (supply capacity) offered by the service $(S)$.

The indicators $T$ and $Q$ were extracted from a survey carried out in the city of Quito in 2014. A total of 231 survey responses were used for this study (error margin $\pm 6,95 \%$ of confidence).

$T$ was measured in hours and $Q$ was measured using a $1-5$ score, where 5 means a person received excellent healthcare attention at their last visit to a primary healthcare service. Cabrera-Barona et al. (2016a) used a categorical score for the indicator $S$, measuring if a person attended a primary healthcare service, a basic hospital, or a specialized hospital. Because this study is applied to primary healthcare services, we used the number of healthcare professionals in the healthcare service.

The indicators were standardized by applying the min-max normalization. The values used to weight the indicators were generated by the application of 
the AHP. The index of healthcare satisfaction $(H C S)$ is calculated by aggregating the weighted normalized indicators. Thus, the equation that describes HCS is as follows:

$$
H C S=0.49(Q)+0.20(S)-0.31(T)
$$

We thereby obtained 231 index values of healthcare satisfaction that were geolocated in the census blocks and census tracts, which could then be linked to the arealevel values of deprivation and healthcare accessibility.

\section{Area-Level Influences of the Indices of Deprivation and Healthcare Accessibility on Individual Healthcare Satisfaction}

Multilevel models were applied to evaluate the index of healthcare satisfaction as a response to the indices of deprivation and healthcare accessibility. These two latter indices represent the context or "neighborhood" that influences individual healthcare satisfaction (Fig. 1).

The individual-level index of healthcare satisfaction is spatially nested in the contexts of deprivation and healthcare accessibility. Multilevel models allow the identification of nested (random) effects when explaining an individual-level (level-1) variable. For the case of this study, a basic two-level model can be applied. This basic model is also named unconditional and it does not include

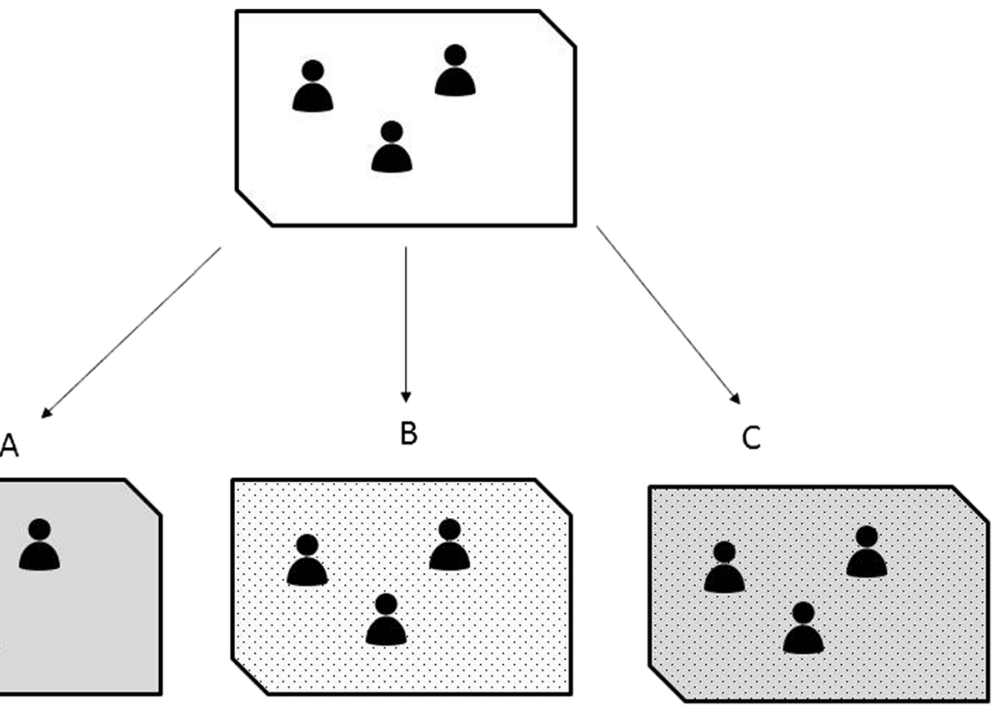

Fig. 1 Individuals are located in a census block or in a census tract. Each one of these individuals has their corresponding healthcare satisfaction index value. The area-level indices of deprivation and healthcare accessibility represent the heath-related contexts of the individuals and influence their healthcare satisfaction. $A$ represents a census block or census tract where the contextual feature is deprivation. $B$ represents the same census block or census tract where the contextual feature is healthcare accessibility. $C$ refers to the census block or census tract with the two contexts: a space where deprivation and healthcare accessibility interact as contextual influences of healthcare satisfaction 
additional individual measures. The level-1 equation of this model is (Nezlek 2007):

$$
Y_{i j}=\beta_{0 j}+r_{i j}
$$

Where $Y_{i j}$ is the level-1 dependent variable in area $j, \beta_{0 j}$ is the level-1 intercept and $r_{i j}$ represents the level-1 error (individual-level residual).

The area-level (level-2) equation of the unconditional model is constructed using the level-1 intercept $\beta_{0 j}$ as the dependent variable of the level-2 equation (Russell 1996):

$$
\beta_{0 j}=\beta_{0}+u_{0 j}
$$

Where $\beta_{0}$ is the overall mean across areas (fixed effect) and $u_{0 j}$ is the effect of area $j$ on the dependent variable $Y_{i j}$. $u_{0 j}$ is a random term at level-2 and can be considered as the level-2 residual.

The combination of the basic level-1 and level-2 equations is called the totally unconditional model (null model) and it is useful to describe the variance of an individual-level variable between areas (Nezlek 2007). We use the variance partition coefficient (VPC) as the measure to evaluate the variances of the individual-level measure (index of healthcare satisfaction) in relation to the area-level measures (healthcare accessibility and deprivation indices). The VPC measures the proportion of the total variance of the individual-level variable that is due to differences between census blocks (or census tracts). The VPC is calculated by dividing the variance of the intercept of the multilevel model by the total variance of the model.

In conclusion, an index value of healthcare satisfaction $i$ in an area $j$ (census block or census tract) with values of healthcare accessibility, or deprivation, or both, can be modeled as:

$$
Y_{i j}=\beta_{0}+u_{0 j}+r_{i j}
$$

Three kinds of multilevel models were then executed: i) one considering only the deprivation index (context of deprivation), ii) a second considering only the healthcare accessibility index (context of healthcare accessibility), and iii) a third one considering both area-level indices (context of deprivation and healthcare accessibility interaction). These three models were calculated for census blocks and for census tracts.

Because both measures of deprivation and healthcare accessibility include an indicator related to distances to primary healthcare services, the assumption of the statistical independence of the area-level measures can be infringed. To ensure this statistical independence, we applied an orthogonal transformation to the index of healthcare accessibility before running the multilevel models.

Finally, to evaluate the significance of areal influences two tests were performed: the likelihood ratio (LR) test and the Wald test. Both tests compare the unconditional model with a null single-level model.

\section{Results}

The city of Quito has low levels of deprivation: the average of the index was $0.13 \pm 0.09 \mathrm{std}$. dev. for the census blocks and $0.17 \pm 0.13 \mathrm{std}$. dev. for the census 
tracts. However, this does not mean the absence of inequalities in the city. Figure 2 depicts the results of the deprivation index at both scales of analyses: census blocks (Fig. 2a) and census tracts (Fig. 2b). It is clear that, independently of the scale, deprivation is concentrated in the peripheries of the city such as in the extreme northwest, the extreme south-east and the extreme south-west.

The index of healthcare accessibility has an average of $0.04 \pm 0.06$ std. dev. for the census blocks and $0.06 \pm 0.07 \mathrm{std}$. dev. for the census tracts. The results of this index must be interpreted cautiously because medium scores of gravity-based models of healthcare accessibility may range around 0.001 (Luo 2014; Luo and Qi 2009). Figure 3 geographically illustrates the index of healthcare accessibility: accessibility

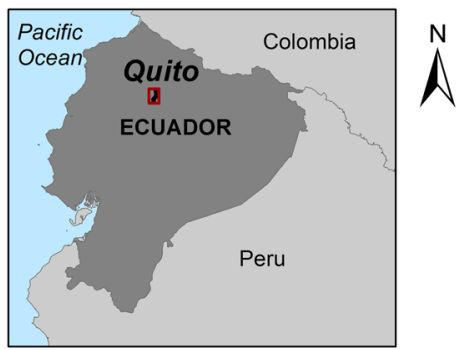

\section{Quito}
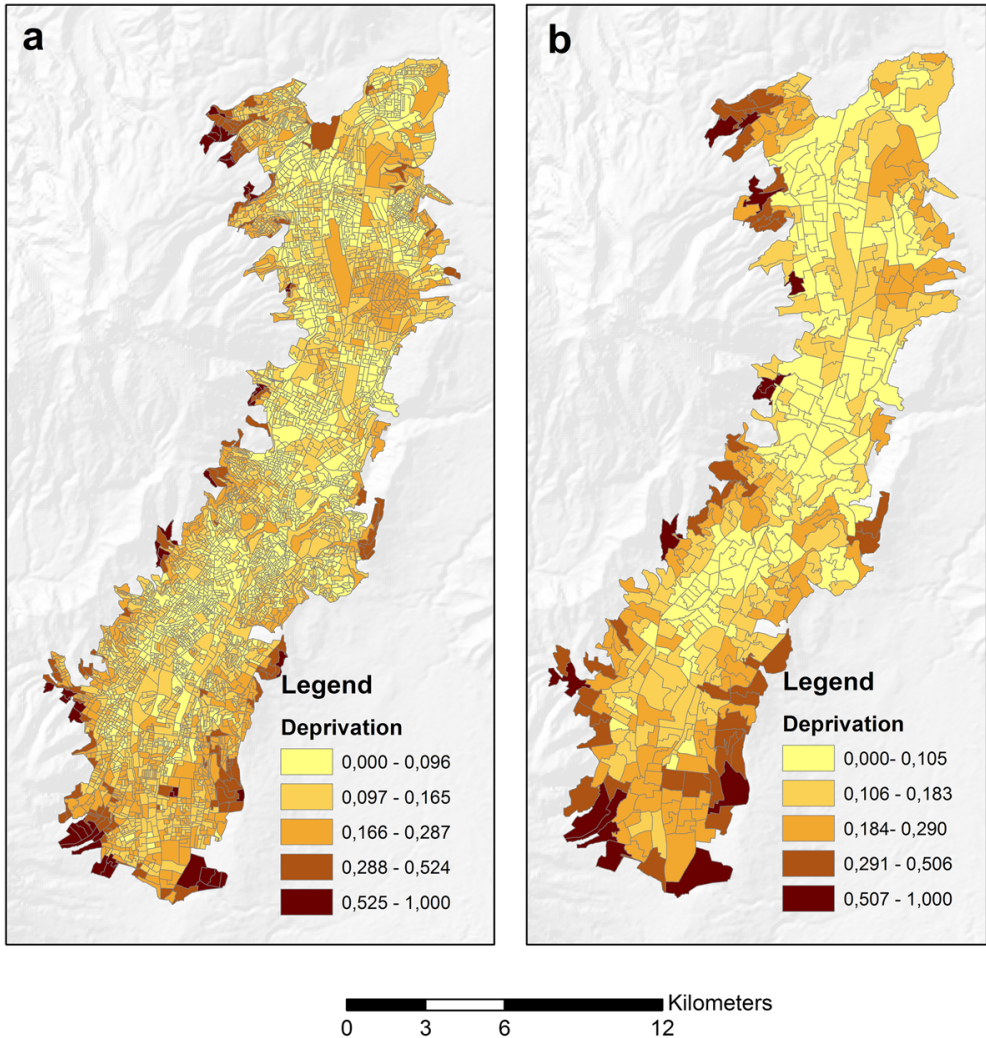

Kilometers

Fig. 2 Deprivation index at census block scale (a) and census tract scale (b) 


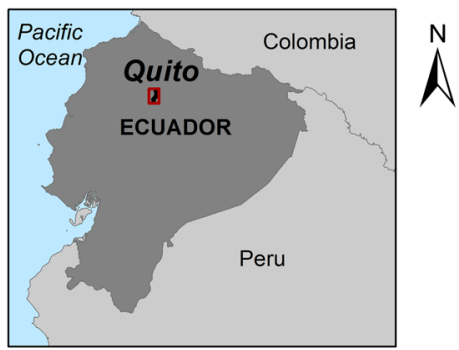

\section{Quito}
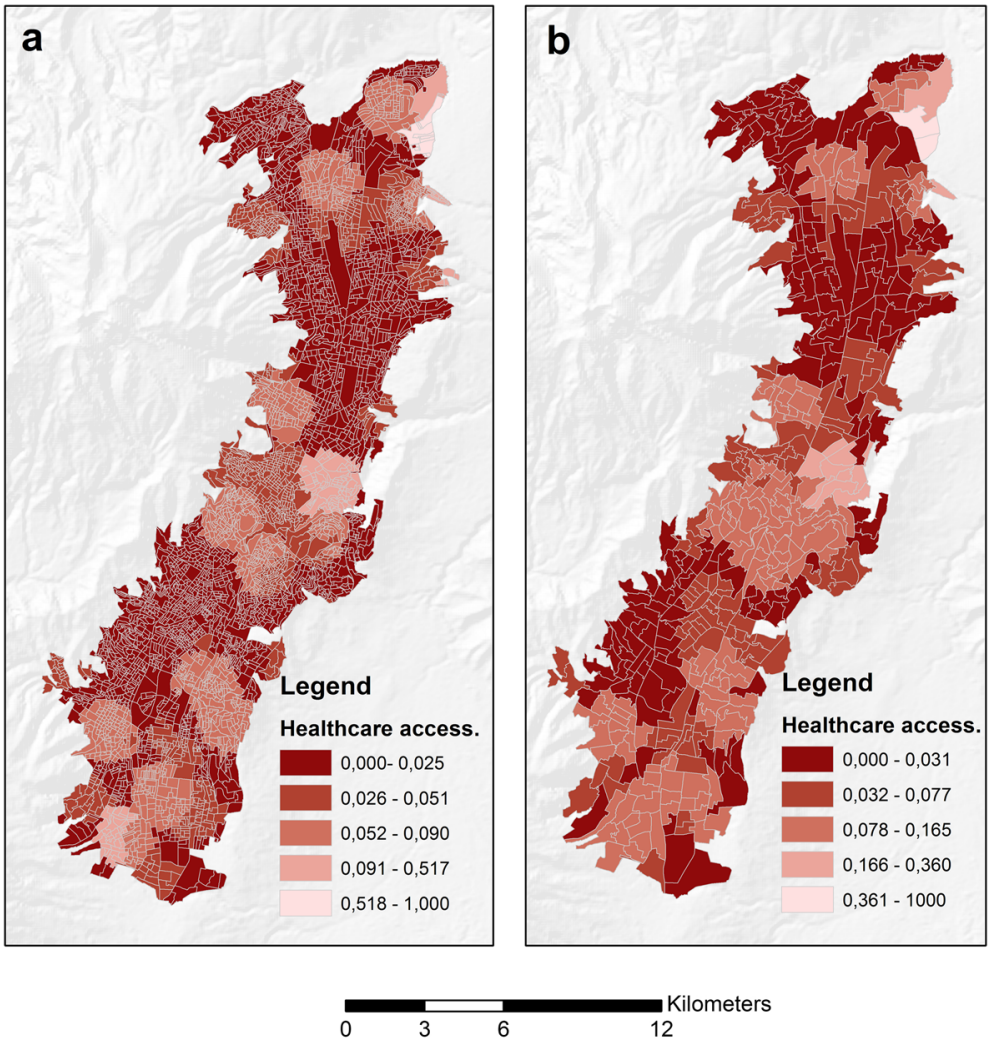

Fig. 3 Index of healthcare accessibility at census block scale (a) and census tract scale (b)

scores higher than 0.001 show that the city of Quito has a good geographical access to healthcare services in most of its census blocks or census tracts. This means that the city's population has a reasonable access to primary healthcare services from a spatial point of view.

The index of healthcare satisfaction has a mean of $0.46 \pm 0.16$ std. dev. Results showed that most interviewed people experienced fair satisfaction with healthcare (satisfaction scores between 0.36 and 0.50 ). Only few people were determined to have low $(<0.30)$ and high $(>0.58)$ healthcare satisfaction: $11 \%$ and $7 \%$ respectively.

Table 4 shows the three types of multilevel models calculated for census blocks as well as for census tracts. The first type of model considers a context of deprivation; the 
Table 4 Results of the multilevel models

\begin{tabular}{|c|c|c|c|c|c|c|c|c|c|}
\hline & Contexts & Intercept & $\begin{array}{l}\text { Std. } \\
\text { errors }\end{array}$ & $\begin{array}{l}\text { Level } 1 \\
\text { variance }\end{array}$ & $\begin{array}{l}\text { Level } 2 \\
\text { variance }\end{array}$ & VPC & LR & $\begin{array}{l}\text { Wald } \\
\text { test }\end{array}$ & AIC \\
\hline \multirow{3}{*}{$\begin{array}{l}\text { Census } \\
\text { blocks }\end{array}$} & Deprivation & 34.04 & 1.46 & 240.35 & 80.31 & 0.25 & 14.60 & 0.0001 & 1979.70 \\
\hline & Healthcare accessibility & 34.51 & 1.95 & 245.70 & 80.30 & 0.25 & 25.61 & 0.0000 & 1968.70 \\
\hline & $\begin{array}{l}\text { Healthcare accessibility } \\
\text { and deprivation }\end{array}$ & 34.01 & 1.39 & 232.32 & 91.67 & 0.28 & 11.07 & 0.0009 & 1983.20 \\
\hline \multirow{3}{*}{$\begin{array}{l}\text { Census } \\
\text { tracts }\end{array}$} & Deprivation & 35.06 & 1.89 & 239.65 & 80.71 & 0.25 & 29.27 & 0.0000 & 1965.00 \\
\hline & Healthcare accessibility & 34.09 & 1.98 & 251.79 & 66.27 & 0.21 & 26.63 & 0.0000 & 1967.70 \\
\hline & $\begin{array}{l}\text { Healthcare accessibility } \\
\text { and deprivation }\end{array}$ & 32.54 & 1.56 & 258.23 & 60.45 & 0.19 & 15.22 & 0.0000 & 1979.10 \\
\hline
\end{tabular}

second type of model considers a context of healthcare accessibility; the third type of model considers both kinds of contexts, deprivation and healthcare accessibility.

The results of the fixed effects of the models are the intercepts and the standard errors. The intercepts are similar for all models and what is interesting is that all the contexts considered are positively related to individual healthcare satisfaction. This is concordant with the low levels of deprivation and high levels of healthcare accessibility of the city. The obtained standard errors mean that the fixed effects are precise considering a $95 \%$ confidence interval.

The random effects are the most interesting part of the multilevel models for this study because it is possible to measure the effects of the area-level deprivation and healthcare accessibility on individual healthcare satisfaction. These effects are summarized in the VPC, LC and Wald test statistics. The obtained variance partition coefficients (VPC) do not significantly vary between the different kinds of contexts and between census blocks and census tracts: $25 \%$ of the variance of individual healthcare satisfaction can be attributed to differences between census blocks or census tracts where deprivation is the area-level feature; $25 \%$ of the variance of individual healthcare satisfaction can be attributed to differences between healthcare accessibility values of census blocks, and $21 \%$ of the variance of this satisfaction can be attributed to differences between healthcare accessibility values of census tracts.

Notwithstanding, a small difference can be identified when the context encompasses both deprivation and healthcare accessibility: in the case of census blocks, $28 \%$ of the variance of healthcare satisfaction can be attributed to differences between census blocks with both characteristics deprivation and healthcare accessibility, while in the case of census tracts this variance is lower, at $19 \%$. The LR and Wald tests show the significance of all these area-level influences on healthcare satisfaction. In the case of the LR test, all the values obtained are higher than 3.84 (the 5\% point of a chi-squared distribution with 1 degree of freedom), which strongly supports the area-level influences of this study. The $p$-values of the Wald test complement the LR test results, and confirm the high significance of the area-level influences at both scales.

Finally, the Akaike information criterion (AIC) showed a similar performance for all the models. The lower AIC corresponds to the model considering the deprivation context at census tract scale. 


\section{Discussion}

In this paper we proposed a methodology to examine scale effects of MAUP and effects of the UGCoP when analyzing the influence of deprivation and healthcare accessibility on healthcare satisfaction. This study demonstrates that there are no marked variations in healthcare satisfaction between the different contexts and different scales of analysis. Therefore, no severe MAUP and UGCoP were found. Interestingly, even when our main focus was to evaluate MAUP and UGCoP effects, we also found striking evidence of area-level influence of deprivation and healthcare accessibility on individual healthcare satisfaction.

The scale effects remained relatively similar when comparing census blocks and census tracts. For example, regarding the context of deprivation, a VPC of 0.25 was found for both census blocks for census tracts. Only the VPCs of the contexts with both healthcare accessibility and deprivation suggest the existence of MAUP.

In the case of the models performed at census blocks scale, the VPCs are similar, which can be interpreted as no evidence of UGCoP. In the case of the census tracts, the VPCs found between the contexts of deprivation and healthcare accessibility mean that there is practically no UGCoP. However, between these contexts and the context where deprivation and healthcare accessibility interact, there is a minimal UGCoP.

The population data to construct the deprivation and the healthcare accessibility indices was obtained from the 2010 Census, and the data to construct the healthcare satisfaction index was obtained in the year 2014. The results of the multilevel models showed a minimal temporal "mismatch" related to the UGCoP in the study area. This minimal temporal mismatch could be ascribed to the use of aggregated data to compose the indices applied, and to the fact that this data was obtained in years relatively close to each other. However, it is more likely to have a day-to-day stability of the dynamics of the geographic contexts. This stability can also be presented during longer time frames, e.g. years. For instance, Wheaton and Clarke (2003) identified a lagged effect of childhood neighborhood disadvantages on early adult mental health. They showed cumulative neighborhoods' effects on individuals. Our findings corroborate this phenomenon.

In terms of health policy making, the results of this study are promising: the levels of healthcare accessibility and deprivation in neighborhoods have a cumulative effect on individuals' healthcare satisfaction. Penchansky and Thomas (1981) determined that multidimensional accessibility to healthcare can be related to the difficulty of a person getting to a healthcare service and to the satisfaction of the received service. This means that accessibility can be divided into two general components: the potential access and realized access (Andersen 1995). Potential access can be measured with gravity-based models (Delamater 2013) and realized access can be measured with healthcare satisfaction. In our study we identified that this potential access to healthcare at neighborhood level may explain satisfaction with the realized access at individual level. Health policy makers and urban planners can pay special attention to the spatial distribution of primary healthcare services in different parts of the city to avoid inequitable access and possibly future low satisfaction with the services.

Deprivation is a contextual or neighborhood-based measure that is useful in explaining health outcomes. Indeed, there is broad experience in showing the relationships of deprivation and different kinds of health outcomes (Boyle et al. 2001; Cabrera- 
Barona et al. 2015; Carstairs 1995; Havard et al. 2008; Lalloué et al. 2013). The importance of studying contextual social disadvantages also lies in the fact that deprived neighborhoods foster negative views or low satisfaction in individuals living there (Gambaro et al. 2015). Local planners and policy makers need to take action in the deprived areas identified in Quito. The reduction of deprivation in these areas is fundamental to reduce not only socio-economic inequalities, but also health inequalities. Thereby, an essential issue is to decide the scale of intervention to reduce deprivation. The spatial stability of our results in both MAUP scale effects and the spatial dimension of the UGCoP can support this decision. The satisfaction with healthcare is not only influenced by the geographical context of the healthcare services locations, but also by the geographical contexts of where people's households are located. Furthermore, the influence is similar at different scales. Health and urban policy makers can thus be aware that healthcare satisfaction may also be influenced by deprivation of the neighborhoods where patients live. They can also choose the reporting unit that best fits their own interests and purposes. In general, health analysts and decision makers tend to choose areas of analysis with the finest spatial resolution, e.g. the census blocks (Cabrera-Barona et al. 2016b). In other cases, selecting larger census areas, e.g. the census tracts, can avoid the difficulty of implementing interventions to very small areas, and can support the formulation of policies for a larger population (Séguin et al. 2012). Additionally, a better understanding of the relationships between social and health measures can be accomplished from analyzing both small and large areas (Nelson and Brewer 2015), as we did in this research.

Even though no severe MAUP and UGCoP were found, it is essential to be aware that intrinsic differences exist when analyzing social indices at different scales and in different geographical contexts. A simple example is the number of people living in an area. Obvious differences of population between census tracts and census blocks exist when analyzing a social index. Schuurman et al. (2007) found significant differences in populations classified by deprivation in census blocks and census tracts. We therefore claim that some health-related processes and relationships may be similar at different scales and contexts, but all oversimplification in the interpretation of statistical results should be avoided, and the MAUP and the UGCoP always need to be monitored to ensure the best possible decision making.

We are aware that this research has some limitations. First, no additional individuallevel explanatory variables were used. Our index of healthcare satisfaction can somewhat overcome this limitation due to its multidimensionality; however, healthcare satisfaction may be influenced by a physician's characteristics (e.g. listening skills), or by a patient's characteristics (e.g. having insurance, car ownership). Healthcare satisfaction can also be influenced by a "healthcare service scale". For instance, it may be possible to score the quality of the primary healthcare service as a whole. In this case, three-level models need to be applied because healthcare satisfaction may be influenced by the quality of the physician's attention, the quality of the service, and the geographical context of where the patient lives. A second limitation is that only census areas were used to evaluate the MAUP and the UGCoP. Even though most measures of deprivation and gravity-based models of healthcare accessibility are calculated in census areas, we highlight that the complex reality represented by these indices is not limited to these kinds of areas. Flowerdew et al. (2008) used census areas as building blocks to construct alternative neighborhoods to understand area-level influence on 
health. We believe that future research can apply the methodology presented in this study to such alternative neighborhoods. These alternative neighborhoods could also be used as an additional level 2 unit in multilevel models, and these models could be compared to different non-hierarchical null models in order to assess scale and zoning effects of the MAUP.

Alternative delineations of neighborhoods have been applied to represent social and ecological indices, including deprivation (Wei et al. 2017). These alternative neighborhoods have been created using different kinds of zoning systems. A comparison of the deprivation index used in this study between different zoning systems may be useful to evaluate the MAUP and the UGCoP. Additionally, independently of possible changes of neighborhood delineation to represent deprivation, it is an important issue to identify possible changes of census indicators at different time-points (Norman 2010). Modifications in these kinds of indicators would affect the construction of a deprivation index and, consequently, would make the multi-temporal evaluations of the MAUP and the UGCoP less comparable.

Future studies could exclude indicators A (percent of the population that have a long-term disability) and $\mathrm{J}$ (distance to the nearest primary healthcare service) when constructing the deprivation index. These indicators already being measures of health, and infringements of statistical independence can take place when relating the deprivation index to the healthcare accessibility index. In this study we overcame this problem by applying orthogonal transformation to the index of healthcare accessibility; however, future deprivation indices could be composed by indicators B to I of Table 1. Removing the health domain from deprivation indices can avoid mathematical coupling, does not dramatically change the measurement of the socioeconomic inequalities in census areas, and may be a better practice when working with deprivation indices (Adams and White 2006).

Future research can also use different kinds of indices of deprivation and healthcare accessibility. The method used to construct the deprivation index can be transferred to other studies. Depending of the reality of the study area, interests of the researchers, and availability of information, different indicators to the ones we used to construct the deprivation index can be used.

It is also recommended that future work uses and compares area-level influences of different measures of healthcare accessibility (e.g. Euclidean distances, healthcare services catchment areas). The method to construct our index of healthcare accessibility can also be transferred to other studies. However, the distances thresholds of the index need to vary with respect to the reality of other study areas. Another parameter that may vary is the function of travel cost. Finally, measures of spatial autocorrelation can be used to describe spatial patterns of area-level effects (Manley et al. 2006). We believe that future work can use indices of local and global autocorrelation not only to evaluate spatial patterns of area-level influences, but also the spatial patterns of the indices used in this study.

\section{Conclusion}

The scale effects of MAUP and the effects of the UGCoP were minimal when analyzing the area-level influence of deprivation and healthcare accessibility on 
healthcare satisfaction. We recommend that analyses of scale and the geographical context should be incorporated in all studies that analyze health phenomena. This study also added a significant piece of evidence of neighborhood effects in health outcomes: a striking area-level influence of deprivation and healthcare accessibility on healthcare satisfaction was determined. We consider this work useful to support policy making, to reduce social and health inequalities in the study area.

Acknowledgements Open access funding provided by Austrian Science Fund (FWF). The presented work has been funded by the Ecuadorian Secretary of Higher Education, Science, Technology and Innovation and the Ecuadorian Institute of Promotion of Human Talent (Scholarship contract No. 375-2012). It has also partially been funded by the Austrian Science Fund (FWF) through the Doctoral College GIScience (DK W 1237 N23) at the University of Salzburg.

Open Access This article is distributed under the terms of the Creative Commons Attribution 4.0 International License (http://creativecommons.org/licenses/by/4.0/), which permits unrestricted use, distribution, and reproduction in any medium, provided you give appropriate credit to the original author(s) and the source, provide a link to the Creative Commons license, and indicate if changes were made.

\section{References}

Adams, J., \& White, M. (2006). Removing the health domain from the index of multiple deprivation 2004effect on measured inequalities in census measure of health. Journal of Public Health, 28(4), 379-383.

Aday, L. A., \& Andersen, R. M. (1974). A framework for the study of access to medical care. Health Services Research, 9, 208-220.

Andersen, R. M. (1995). Revisiting the behavioral model and access to medical care: does it matter? Journal of Health and Social Behavior, 36, 1-10.

Bell, N., Schuurman, N., Oliver, L., \& Hayes, M. (2007). Towards the construction of place-specific measures of deprivation: a case study from the Vancouver metropolitan area. The Canadian Geographer, 51(4), $444-461$.

Bissonnette, L., Wilson, K., Bell, S., \& Shah, T. (2012). Neighbourhoods and potential access to healthcare: the role of spatial and aspatial factors. Health \& Place, 18, 841-853.

Bleich, S. N., Özaltin, E., \& Murray, C. J. L. (2009). How does satisfaction with the healthcare system relate to patient experience? Bulletin of the World Health Organization, 87, 271-278.

Boroushaki, S., \& Malczewski, J. (2008). Implementing an extension of the analytical hierarchy process using ordered weighted averaging operators with fuzzy quantifiers in ArcGIS. Computers \& Geosciences, 34, 399-410.

Boyle, P., Gatrell, A., \& Duke-Williams, O. (2001). Do area-level population change, deprivation and variations in deprivation affect individual-level self-reported limiting long-term illness? Social Science \& Medicine, 53, 795-799.

Briant, A., Combes, P. P., \& Lafourcade, M. (2010). Dots to boxes: do the size and shape of spatial units jeopardize economic geography estimations? Journal of Urban Economics, 67, 287-302.

Butler, D., Petterson, S., Phillips, R. L., \& Bazemore, A. W. (2013). Measures of social deprivation that predict health care access and need within a rational area of primary care service delivery. Health Services Research, 48(2), 539-559.

Cabrera-Barona, P., Murphy, T., Kienberger, S. \& Blaschke, T. (2015) A multi-criteria spatial deprivation index to support health inequality analyses. International Journal of Health Geographics, 14(11).

Cabrera-Barona, P., Blaschke, T. \& Kienberger, S. (2016a) Explaining accessibility and satisfaction related to Healthcare: A Mixed-Methods Approach. Social Indicators Research doi:10.1007/s11205-016-1371-9.

Cabrera-Barona, P., Wei, C., \& Hagenlocher, M. (2016b). Multiscale evaluation of an urban deprivation index: implications for quality of life and healthcare accessibility planning. Applied Geography, 70, 1-10.

Carstairs, V. (1995). Deprivation indices: their interpretation and use in relation to health. Journal of Epidemiology and Community Health, 49(2), S3-S8.

Chen, Y., Yu, J., \& Khan, S. (2010). Spatial sensitivity analysis of multi-criteria weights in GIS-based land suitability evaluation. Environmental Modelling and Software, 25, 1582-1591. 
Crooks, V., \& Schuurman, N. (2012). Interpreting the results of a modified gravity model: examining access to primary health care physicians in five Canadian provinces and territories. BMC Health Services Research, 12(230), 1-13.

Delamater, P. (2013). Spatial accessibility in suboptimally configured healthcare systems: a modified two-step floating catchment area (M2SFCA) metric. Health \& Place, 24, 30-43.

Diez-Roux, A. V., \& Mair, C. (2010). Neighborhood and health. Annals of the New York Academy of Sciences, $1186,125-145$.

Dumedah, G., Schuurman, N., \& Yang, W. (2008). Minimizing effects of scale distortion for spatially grouped census data using rough sets. Journal of Geographical Systems, 10, 47-69.

Flowerdew, R. (2011). How serious is the modifiable areal unit problem for analysis of English census data? Population Trends, 145, 1-13.

Flowerdew, R., Manley, D. J., \& Sabel, C. E. (2008). Neighbourhood effects on health: does it matter where you draw the boundaries? Social Science \& Medicine, 66, 1241-1255.

Fotheringham, A. S., \& Wong, D. W. S. (1991). The modifiable areal unit problem in multivariate statistical analysis. Environment \& Planning A, 23, 1025-1044.

Gambaro, L., Joshi, H., Lupton, R., Fenton, A., \& Lennon, M. C. (2015). Developing better measures of Neighbourhood characteristics and change for use in studies of residential mobility: a case study of Britain in the early 2000s. Applied Spatial Analysis and Policy. doi:10.1007/s12061-015-9164-0.

Guagliardo, M. (2004). Spatial accessibility of primary care: concepts, methods and challenges. International Journal of Health Geographics, 3, 1-13.

Hagenlocher, M., Kienberger, S., Lang, S., \& Blaschke, T. (2014). Implications of spatial scales and reporting units for the spatial modelling of vulnerability. In R. Vogler, A. Car, J. Strobl, \& D. Griesebner (Eds.), GI Forum 2014. Geospatial innovation for society (pp. 197-206). Wien: ÖAW Verlag.

Hansen, W. G. (1959). How accessibility shapes land use. Journal of the American Planning Institute, 25, 7376.

Havard, S., Deguen, S., Bodin, J., Louis, K., Laurent, O., \& Bard, D. (2008). A small-area index of socioeconomic deprivation to capture health inequalities in France. Social Science \& Medicine, 67, 2007-2016.

Haynes, R., \& Gale, S. (2000). Deprivation and poor health in rural areas: inequalities hidden by averages. Health \& Place, 6, 275-285.

Hekkert, K. D., Cihangir, S., Kleefstra, S. M., van den Berg, B., \& Kool, R. B. (2009). Patient satisfaction revisited: a multilevel approach. Social Science and Medicine, 69, 68-75.

Hiscock, R., Pearce, J., Blakely, T., \& Witten, K. (2008). Is neighborhood access to health care provision associated with individual-level utilization and satisfaction? Health Services Research, 43, 2183-2200.

Huff, D. L. (1963). A probabilistic analysis of shopping center trade areas. Land Economics, 39, 81-90.

Joseph, A. E., \& Bantock, P. R. (1982). Measuring potential physical accessibility to general practitioners in rural areas: a method and case study. Social Science and Medicine, 16, 85-90.

Joseph, A. E., \& Phillips, D. R. (1984). Accessibility and utilization: geographical perspectives on health care delivery. New York: Harper and Row.

Kwan, M. (2012a). The uncertain geographic context problem. Annals of the Association of American Geographers, 102(5), 958-968.

Kwan, M. (2012b). How GIS can help address the uncertain geographic context problem in social science research. Annals of GIS, 18(4), 245-255.

Lalloué, B., Monnez, J. M., Padilla, C., Kihal, W., Le Meur, N., Zmirou-Navier, D., \& Deguen, S. (2013). A statistical procedure to create a neighborhood socioeconomic index for health inequalities analysis. International Journal for Equity in Health, 12(21), 1-11.

Luo, J. (2014). Integrating the huff model and floating catchment area methods to analyze spatial access to healthcare services. Transactions in GIS, 18(3), 436-448.

Luo, W., \& Qi, Y. (2009). An enhanced two-step floating catchment area (E2SFCA) method for measuring spatial accessibility to primary care physicians. Health \& Place, 15, 1100-1107.

Manley, D., Flowerdew, R., \& Steel, D. (2006). Scales, levels and processes: studying spatial patterns of British census variables. Computers, Environment and Urban Systems, 30, 143-160.

Mideros, A. (2012). Ecuador: defining and measuring multidimensional poverty, 2006-2010. CEPAL Review, 108, 49-67.

Nelson, J. K., \& Brewer, C. A. (2015). Evaluating data stability in aggregation structures across spatial scales: revisiting the modifiable areal unit problem. Cartography and Geographic Information Science, O(0), 116.

Nezlek, J. (2007). A multilevel framework for understanding relationships among traits, states, situations and behaviours. European Journal of Personality, 21, 789-810. 
Norman, P. (2010). Identifying change over time in small area socio-economic deprivation. Applied Spatial Analysis and Policy, 3(2), 107-138.

Openshaw, S. (1984). The modifiable areal unit problem. Norwich: GeoBooks. CATMOG, 38.

Organisation for Economic Co-operation and Development (2008). Handbook on Constructing Composite Indicators: Methodology and User Guide. www.oecd.org/std/42495745.pdf.

Pampalon, P., Hamel, D., Gamache, P., \& Raymond, G. (2009). A deprivation index for health planning in Canada. Chronic Diseases in Canada, 29(4), 178-191.

Pascoe, G. C. (1983). Patient satisfaction in primary health care. Evaluation and Program Planning, 6, 185210.

Pasetto, R., Sampaolo, L., \& Pirastu, R. (2010). Measures of material and social circumstances to adjust for deprivation in small-area studies of environment and health: review and perspectives. Annali dell Istituto Superiore di Sanita, 46(2), 185-197.

Penchansky, R., \& Thomas, J. W. (1981). The concept of access: definition and relationship to consumer satisfaction. Medical Care, 19(2), 127-140.

Pietrzak, M. B. (2014). The modifiable areal unit problem-analysis of correlation and regression. Equilibrium. Quarterly Journal of Economics and Economic Policy, 9(3), 113-131.

Russell, E. (1996). A multilevel modelling approach to examining the effects of area of residence on health and functioning. Journal of the Royal Statistical Society: Series A (Statistics in Society), 159(1), 61-75.

Saaty, T. L. (1977). A scaling method for priorities in hierarchical structure. Journal of Mathematical Psychology, 15(3), 34-39.

Saaty, R. W. (1987). The analytic hierarchy process-what it is and how it is used. Mathematical Modelling, 9(3-5), 161-176.

Schuurman, N., Bell, N., Dunn, J. R., \& Oliver, L. (2007). Deprivation indices, population health and geography: an evaluation of the spatial effectiveness of indices at multiple scales. Journal of Urban Health: Bulletin of the New York Academy of Medicine, 84(4), 591-603.

Schuurman, N., Bérubé, M., \& Crooks, V. A. (2010). Measuring potential spatial access to primary health care physicians using a modified gravity model. The Canadian Geographer, 54(1), 29-45.

Séguin, A. M., Apparicio, P., \& Riva, M. (2012). The impact of geographical scale in identifying areas as possible sites for area-based interventions to tackle poverty: the case of Montréal. Applied Spatial Analysis and Policy, 5, 231-251. doi:10.1007/s12061-011-9068-6.

Senior, M. L. (1991). Deprivation payments to GPs: not what the doctor ordered. Environment and Planning. C, Government \& Policy, 9, 79-94.

Soobader, M., LeClere, F., Hadden, W., \& Maury, B. (2001). Using aggregate geographic data to proxy individual socioeconomic status: does size matter? American Journal of Public Health, 91, 632-636.

Townsend, P. (1987). Deprivation. Journal of Social Policy, 16, 125-146.

Townsend, P., Phillimore, P., \& Beattie, A. (1988). Health and deprivation: inequality and the north. London: Croom Helm.

Wan, N., Zhan, F. B., Zou, B., \& Wilson, G. (2013). Spatial access to health care services and disparities in colorectal cancer stage at diagnosis in Texas. The Professional Geographer, 65(3), 527-541.

Wei, C., Padgham, M., Cabrera-Barona, P., \& Blaschke, T. (2017). Scale-free relationships between social and landscape factors in Urban Systems. Sustainability, 9(84), 1-19.

Wheaton, B., \& Clarke, P. (2003). Space meets time: integrating temporal and contextual influences on mental health in early adulthood. American Sociological Review, 68(5), 680-706. 